\title{
ALFVÉN INSTABILITY AND MICROMAGNETIC ISLANDS IN A PLASMA WITH SHEARED MAGNETIC FIELDS
}

BY

J. HSU, P. KAW, AND LIU CHEN

\section{PLASMA PHYSICS LABORATORY}
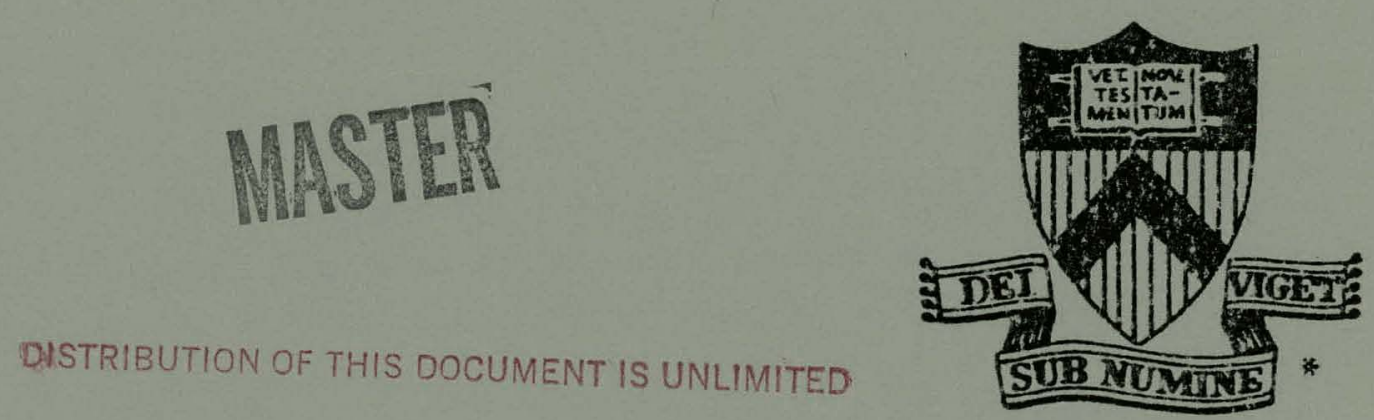

This work was supporterl by U. S. Energy Kesearch and Development Administration Contract EY-76-C-02-3073. Reproduction, translation, publication, use and disposal, in whole or in part, by or for the United States Government is permitted. 


\section{DISCLAIMER}

This report was prepared as an account of work sponsored by an agency of the United States Government. Neither the United States Government nor any agency Thereof, nor any of their employees, makes any warranty, express or implied, or assumes any legal liability or responsibility for the accuracy, completeness, or usefulness of any information, apparatus, product, or process disclosed, or represents that its use would not infringe privately owned rights. Reference herein to any specific commercial product, process, or service by trade name, trademark, manufacturer, or otherwise does not necessarily constitute or imply its endorsement, recommendation, or favoring by the United States Government or any agency thereof. The views and opinions of authors expressed herein do not necessarily state or reflect those of the United States Government or any agency thereof. 


\section{DISCLAIMER}

Portions of this document may be illegible in electronic image products. Images are produced from the best available original document. 
This report was prepared as an account of work sponsored by the United States Government. Neither the United States nor the United States Energy Research and Development Administration, nor any of their employees, nor any of their contractors, subcontractors, or their employees, makes any warranty, express or implied, or assumes any legal liability or responsibility for the accuracy, completeness or usefulness of any information, apparatus, product or process disclosed, or represents that its use would not infringe privately owned rights.

Printed in the United States of America. Available from

National Technical Information Service

U. S. Department of Commerce 5285 Port Royal Road Springfield, Virginia 22151

Price: Printed Copy $\$+$; Microfiche $\$ 3.00$

$$
\begin{gathered}
* \text { Pages } \\
1-50 \\
51-150 \\
151-325 \\
326-500 \\
501-1000
\end{gathered}
$$

NTIS

Selling Price

$\$ 4.00$

5.45

7.60

10.60

13.60 
Alfvén Instability and Micromagnetic Islands in a Plasma with Sheared Magnetic Fields

J. Hsu, P. Kaw, and Liu Chen

Princeton Plasma Physics Laboratory

Princeton University, Princeton, N. J. 08540 
Alfvẹn Instability and Micromagnetic Islands in a Plasma with Sheared Magnetic Fields*

\author{
J. Hsu, P. Kaw, and Li'u Chen \\ Princeton University Plasma Physics Laboratory \\ Princeton, New Jersey 08540
}

\title{
ABSTRACT
}

The normal mode equation for coupled drift and Alfvén waves in a finite- $\beta$ nonuniform plasma with a sheared magnetic field is solved, in the slab geometry, to investigate the instability of slow Alfvén waves. It is shown, that, besides having an appreciable growth rate, the instability also produces microscopic "tearing" of the rational surfaces which has important implications for anomalous transport. 
Traditionally, anomalous transport in magnetically confined plasmas is ascribed to the presence of electrostatic drift-wave fluctuations, which are driven to large amplitudes by the expansion free energy associated with density and temperature gradients in the plasma. A mechanism of equally great interest, which seems to have received much less attention, is the excitation of lowErequency, short wavelength, predominantly electromagnetic fluctuations by the same free energy source. These two kinds of fluctuations can produce loss of confinement by fundamentally different processes. The former is limited to an enhancement of transports across the magnetic surfaces. The latter, on the other hand (in the presence of dissipation), may produce local "tearing" and break-up of magnetic surfaces and thus essentially permit transports along newly "re-connected" field lines. This proccss is especially significant for short-wavelength fluctuations, since the moderational surfaces are densely packed and considerable overlap of "magnetic islands" may readily occur.

In this letter, we examine the instabi.tity of slow Alfvén waves in a finite- $\beta$ nonuniform plaoma with sheared llagnet1c fields. The local dispersion relation for coupled drift and Alfvén waves (which is relevant to the shear-free case) is well known, ${ }^{2}$ viz.

$$
k_{y}^{2} \lambda_{s}^{2}+\left(1-\frac{\omega^{2}}{\omega_{A}^{2}}\right)\left(1-\frac{\omega_{e}^{*}}{\omega}\right)(1+i \delta \omega)=0
$$

where $\lambda_{s}^{2}=T_{e} / M_{\omega} \omega_{c i}^{2}, \omega_{A}=k_{f} v_{A}, \omega_{e}^{*}=-\left(k_{Y} c T_{e} / e_{0}\right)\left(1 / n_{0}\right)\left(d n_{0} / d x\right)$ with $-e<0$ and $\delta>0$ represents the dissipation effect on electrons. We have neglected the ion-sound and ion damping terms. 
Equation (1) exhibits two instabilities:

$$
\begin{aligned}
& \omega_{1} \simeq \omega_{e}^{*}+\omega_{e}^{*} k_{y}^{2} \lambda_{S}^{2}\left(1-i \omega_{e}^{*} \delta\right) /\left(\omega_{e}^{* 2} / \omega_{A}^{2}-1\right) \\
& \omega_{2} \simeq \omega_{A}-\omega_{A} k_{Y}^{2} \lambda_{S}^{2}\left(1-i \omega_{A} \delta\right) / 2\left(\omega_{e}^{*} / \omega_{A}-1\right) .
\end{aligned}
$$

For $\omega_{e}^{*}<\omega_{A}, \omega_{1}$ is unstable. This is the usual electrostatic drift instability. In this case $\omega_{2}$, which corresponds to slow Alfvén waves, is stable. For $\omega_{e}^{*}>\omega_{A}$, the Alfvên root $\omega_{2}$ is unstable and the usual drift wave is stabilized. Note that the introduction of $\omega_{A}$ terms in $\mathrm{Eq}$. (I) can be considered as a finite- $\beta$ effect because $\omega_{A} / \omega_{e}^{*} \rightarrow \infty$ when $\beta \rightarrow 0$.

The normal mode equations for coupled drift and Alfvén waves in the presence of magnetic shear has been investigated previously. ${ }^{3}$ However, this calculation restricts itself to an investigation of the finite- $\beta$ stabilization of the usual electrostatic drift branch $\omega_{1}$. Nobody seems to have recovered the branch $\omega_{2}$ from a nonlocal trcatment, so far. It is this more that we shall be discussing below.

Consider an inhomogeneous (gradient along $\hat{e}_{x}$ ) plasma in a sheared magnetic field $\underset{\sim}{\mathrm{B}}=\mathrm{B}_{0}\left(\hat{\mathrm{e}}_{z}+\hat{e}_{y} \mathrm{x} / \mathrm{L}_{s}\right)$. We describe the electrons by a drift kinetic equation with a krook-type density conserving collision operator. Ions are fully kinetic and collisionless. Since $B=8 \pi n_{0}\left(T_{e}^{:}+T_{i}\right) / B_{0}^{2} \ll I$ and we restrict our attention to slow Alfvén waves which only "bend" the field Iines, the field perturbations can be described in terms of two scalar potentials $\psi, \phi$ defined by 


$$
\delta E_{\|}=-\nabla_{\|} \psi ; \quad \underset{\sim}{E_{\perp}}=-\nabla_{\perp} \phi .
$$

$\psi, \phi$ are related to the vector potential $\mathrm{A} \hat{e}_{z}$ (defined by $\delta B_{1}=\nabla \times \underset{\sim}{A}$ ) through the relation $\psi=\phi-\omega A / k_{\|} c$. Linearizing the kinetic equations, solving for $\delta f$, integrating over the velocity space and using the quasineutrality condition $\delta n_{e} \simeq \delta n_{i}$, we obtain the equation

$$
\lambda^{2} \nabla_{\perp}^{2} \phi=\varepsilon(\omega, x) \psi
$$

where $\lambda^{2}=\lambda_{s}^{2}(I+\tau) \frac{\omega 1 \omega_{e}^{*} \tau}{\omega-\omega_{e}^{*}}, \tau=\frac{T_{i}}{T_{e}}, \lambda_{s}^{2}=\frac{T_{e}}{M \omega_{c i}^{2}}$,

$$
\begin{aligned}
& \varepsilon(\omega, x)=1+\left[\frac{\omega}{\left|k_{\|} v_{e}\right|} z\left(\frac{\omega+i v_{e}}{\left|k_{\|} v_{e}\right|}\right)\right]\left[1+\frac{i v_{e}}{\left|k_{q} v_{e}\right|} z\left(\frac{\omega+i v_{e}}{\left|k_{\|} v_{e}\right|}\right)\right]^{-1} \\
& +i \pi^{1,2} \tau \frac{\omega+\omega_{e^{*}}^{\star}}{\omega-\omega_{e}^{*}} \frac{x_{I}}{x} \exp \left(=\frac{x_{T}^{2}}{x^{2}}\right)-\frac{x^{2}}{x_{T}^{2}}
\end{aligned}
$$

$k_{\|}=k_{y} x / I_{s} ; x_{I} \equiv \omega I_{s} / k_{y} v_{i}, x_{T}^{2}=\omega^{2} I_{s}^{2}\left(\omega-\omega_{e}^{*}\right) / k_{y}^{2} c_{s}^{2}\left(\omega+\omega_{e}^{*} \tau\right)$ and $\mathrm{z}$ is the plasma dispersion function. We have assumed that for ions, $\left|\omega / k_{1} v_{i}\right|>1$ is satisfied in the whole x-region of interest and that $\left|\lambda_{s}^{2} \tau \nabla_{\perp}^{2}\right| \ll 1$. obtaining the perturbed $\delta j_{\|}$from the first moments of $\delta f_{e, i}$ and using the Ampere's law we obtain a second equation between $\psi$ and $\phi$ viz.

$$
\frac{1}{k_{1}} \nabla_{\perp}^{2}\left[k_{\|}(\psi-\phi)\right]=-\frac{\omega\left(\omega+\omega_{e}^{*} \tau\right)}{\omega_{A}^{2}} \frac{1}{n_{0}} \nabla_{\perp} \cdot\left(n_{0} \nabla_{\perp} \phi\right)
$$


where $\omega_{A}^{2}=k_{y}^{2} v_{A}^{2}, v_{A}$ being the Alfvén speed. Equations (4) and (6) may be readily combined to give a fourth order differential equation in $\phi$. We now assume $\left|d^{2} / d x^{2}\right| \gg\left|k_{y}^{2}\right|$. Multiplying the fourth order equation by $x^{2}$ and integrating once, we obtain a second order differential equation for $E=d \phi / d x$ :

$$
\lambda^{2} \frac{d}{d x}\left(\frac{d E / d x}{\varepsilon(\omega, x)}\right)-\left(1-\frac{x_{A}^{2}}{x^{2}}\right) E=\frac{C}{x^{2}}
$$

where $x_{A}^{2}=\omega\left(\omega+\omega_{e}^{*} \tau\right) L_{s}^{2} / k_{y}^{2} V_{A}^{2}$ and $C$ is the constant of integration. The physical meaning of this constant can be understood by passing to the large $x$ limit; the two dominant terms are $E+c / x^{2} \simeq 0$ which gives $\delta B_{x} \simeq$ constant. This is the "constant- $\Psi$ " solution in usual resistive instability theories ${ }^{5}$, which then matches on to the outside long-wavelength M̀HD solutions. The mode of present interest can be found without outside long-wavelength MHD support. So we let $C=0$.

The mode-structure of the usual electrostatic drift branch is determined by the $x_{T}$ term in $\varepsilon(\omega, x)$ so that one has to do the ordering $\lambda \sim \mathrm{x}_{\mathrm{T}} \gg \mathrm{x}_{\mathrm{A}}$. Note that $\lambda \sim \mathrm{x}_{\mathrm{T}}$ gives $\left(\omega-\omega_{*}\right) \cdots \omega_{*} L_{\mathrm{n}} / \mathrm{L}_{\mathrm{s}}$, the well-known arift wave result. Note also that $x_{T} / x_{A}>I$ is always satisfied as long as $\omega / \omega_{*}>B$. On the other hand, to study the Alfvén branch, one has to order $\lambda \sim \mathrm{x}_{\mathrm{A}} \ll \bar{x}_{\mathrm{T}}$ so that the mode-structure is decided by the Alfvén term. It is this lack of proper ordering, which has prevented earlier workers ${ }^{3}$ from recovering the Alfven root. Note that $\lambda \sim \mathrm{x}_{\mathrm{A}}$ yields $w \sim k_{y}^{2} v_{A}^{2}\left(\lambda \frac{2}{s} / L_{s}^{2}\right) / w_{*}<\omega_{*}$ as the root. 
To the lowest order then, the Alfven mode is simply described by the equation

$$
\lambda^{2} \frac{d^{2}}{d x^{2}} E=\left(1-\frac{x_{A}^{2}}{x^{2}}\right) E
$$

In this limit, $x_{T}$ and $x_{I}$ are ordered large $(\rightarrow \infty)$ and the electron inertia and electron dissipation are neglected. The solutions are given by $x^{1 / 2} J_{n}(i x / \lambda)$ and $x^{1 / 2} Y_{n}(i x / \lambda)$ where $J_{n}$ and $x_{n}$ are the Bessel functions. The lowest order dispersion relation is yiven by

$$
\left(\frac{\mathrm{x}_{\mathrm{A}}^{2}}{\lambda^{2}}\right)_{0}=\frac{1}{4}-\mathrm{n}^{2}=\frac{\omega_{0}\left(\omega_{0}-\omega_{e}{ }^{k}\right)}{\omega_{\mathrm{A}}^{2}} \frac{\mathrm{L}_{\mathrm{s}}^{2}}{\lambda_{\mathrm{s}}^{2}}
$$

where $\mathrm{n}$ is an integer. The eigenfrequencies are given by

$$
\omega_{0,1} \simeq \omega_{e}^{\star}\left[1+\left(\frac{1}{4}-n^{2}\right) \frac{\omega_{\mathrm{A} O}^{2}}{{ }^{\star}{ }_{e}} \frac{\lambda_{s}^{2}}{L_{s}^{2}}\right]
$$

and

$$
\omega_{0,2} \simeq\left(n^{2}-\frac{1}{4}\right) \frac{\omega_{A 0}^{2}}{\omega_{e}^{t}} \frac{\lambda_{s}^{2}}{L_{s}^{2}} .
$$

The second root corresponds to the Alfvén branch and is the only unstable branch as shown below. To construct outward propagating (or decaying) solutions far away, we have to include combinations of $\mathrm{J}_{\mathrm{n}}$ and $\mathrm{Y}_{\mathrm{n}}$ functions. However, the $\mathrm{Y}_{\mathrm{n}}$ solution blows up at $x=0$. This pathology of the zeroth order eigenfunction is associated with the absence, in the zeroth order equations, of a "dissipative" force which may balance the parallel electric fields 
generated at $\mathbf{x}=0$ by time-dependent magnetic fluctuations. In a collisional plasma, resistivity provides such a force whereas in the collisionless problem, electron inertia takes up that role. We now show that it is possible to remove this pathology by a proper matching solution near $x=0$.

We first consider the collisional case $v_{e}>\omega$ and $v_{e} /\left|k_{\|}\right| v_{e}$ $>1$ in the $x$-region of interest. The equation we want to solve is

$$
\lambda^{2} \frac{d}{d x}\left[\left(1-\frac{x_{R}^{2}}{x^{2}}\right) \frac{d E}{d x}\right]-\left(1-\frac{x_{A}^{2}}{x^{2}}\right) E=0
$$

where $x_{R}$ is the resistive layer thickness defined by $x_{R}^{2}=i \omega v_{e}{ }^{2} /$ $k_{y}^{2} v_{e}^{2}$. We assume $\lambda \sim x_{A}$ and treat $x_{R} / x_{A} \ll 1$ as a small parameter. Introducing $s=i x / \lambda$ as a new variable, the lowest order solution in the outer region, describing outgoing waves, is given by

$$
E^{(0)}=C_{1} s^{1 / 2} H_{V}^{(2)}(s)
$$

where $H_{V}^{(2)}$ is the Hankel function of second kind and

$$
v^{2}=(1 / 4)-\left(x_{A}^{2} / \lambda^{2}\right)
$$

The first order equation in the outer region can be solved with the boundary condition $\mathrm{E}^{(1)}(s \rightarrow m)=0$ : 


$$
\begin{aligned}
& E^{(1)}=\frac{C_{I}}{W}\left[s^{1 / 2} J_{v}(s) \int_{\infty}^{s} \frac{d}{d s^{\prime}}\left(s^{\prime 1 / 2} \underline{J}_{\nu}\right) \frac{d}{d s^{\prime}},\left(s^{\prime 1 / 2} H_{\nu}^{(2)}\right) \frac{x_{R}^{2} / \lambda^{2}}{s^{\prime 2}} d s^{\prime}\right. \\
& \left.-s^{I / 2} \underline{J}_{v}(s) \int_{\infty}^{s} \frac{d}{d s^{\prime}}\left(s^{1 / 2} J_{v}\right) \frac{d}{d s^{\prime}} \cdot\left(s^{\prime I / 2}{ }_{H}^{(2)}\right) \frac{x_{R}^{2} / \lambda^{2}}{s^{\prime 2}} d s^{\prime}\right]
\end{aligned}
$$

where the Wronskian $W=-2 \sin \pi \nu / \pi$. In the inner region, we introduce $\tilde{x}=x / x_{R}$ as a variable and write the lowest order equa tion as

$$
\frac{d}{d \tilde{x}}\left[\left(1-\frac{1}{\tilde{x}^{2}}\right) \frac{d E}{d \tilde{x}}\right]=-\frac{x_{A}^{2} / \lambda^{2}}{\tilde{x}^{2}} E .
$$

Making the transformation $\Phi=\left[\left(\tilde{x}^{2}-1\right) / \tilde{x}\right](d E / d \tilde{x})$, we get an equation which has the solution

$$
\Phi=\left(1-\tilde{\mathrm{x}}^{2}\right)^{1 / 2}\left(\mathrm{C}_{3} \mathrm{P}_{\nu+1 / 2}^{1}(\tilde{\mathrm{x}})+\mathrm{c}_{4} \mathrm{Q}_{\nu+1 / 2}^{1}(\tilde{\mathrm{x}})\right)
$$

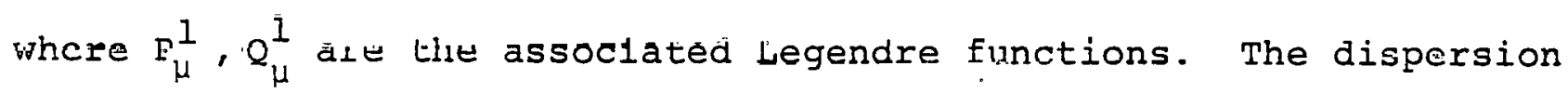
relation is obtained by imposing the parity conditions at $\mathrm{x}=0$ (viz. $\Phi^{\prime}(0)=0$ for even parity and $\Phi(0)=0$ for odd parity), taking the asymptotic expansion of the Legendre functions $(|\tilde{x}| \rightarrow \infty)$ and matching the resultant solution E to small - "s" limits of the outer region solution $E^{(0)}+E^{(1)}$. The dispersion relation takes the rather simple form

$$
\nu^{2}=n^{2}-\frac{1}{2} \frac{x_{R}^{2}}{\lambda^{2}}=\frac{1}{4}-\frac{x_{A}^{2}}{\lambda^{2}}
$$

Equation (16) differs from the zeroth order Eq. (9) only in the first order correction term $\sim x_{R}^{2} / \lambda^{2}$. This result can also be 
obtained by a regular perturbation treatment. Equation (16) can be solved to give an Alfvén root with a frequency $\omega_{0,2}<\omega_{*}$ [defined in Eq. (10)] and a growth rate $=(\mathrm{m} / \mathrm{M})\left(\nu_{\mathrm{ei}} / 2 \beta\right)$. Note that this growth rate has an upper limit $\sim v_{e i} / 2$ (because $\beta \geq \mathrm{m} / \mathrm{M}$ ) and is larger by a factor $\beta^{-1}$ from that of dissipative drift waves. Furthermore, unlike drift waves, there is no shear damping term involved because the wave function is localized deep inside the sound turning point. Note also that for even parity modes $\left.\delta B_{x}\right|_{x=0} \neq 0$ i.e., the magnetic field perturbation has a tearing component at the rational surface. The instability will thus lead to the formation of microscopic magnetic islands on moderational surfaces.

The normal mode equation for the collisionless case $\omega>\nu_{e}$ is

$$
\lambda^{2} \frac{d}{d x}\left(\frac{d E / d x}{1+x_{e} z\left(x e^{/|x|) /|x|}\right.}\right)-\left(1-\frac{x_{A}^{2}}{x^{2}}\right) E=0
$$

where $x_{e}=\omega I_{s} / k_{y} v_{e}$. Assuming $x_{e} \ll x_{A}$, the outer region equation is the same as before. The inner region equation is harder to solve in this case. However, an approximate dispersion relation can be obtained by a regular perturbation theory. It is of the form

$$
v^{2}=1 / 4-x_{A}^{2} / \lambda^{2} \simeq n^{2}-i n \pi^{-1 / 2}\left(x_{e} / \lambda\right) \ln \left(x_{I} / \lambda\right)
$$

Equation (18) differs from Eq. (16) in the form of the perturbation term $\left(x_{e} / \lambda\right) \ln \left(x_{I} / \lambda\right)$ replacing $x_{R}^{2} / \lambda^{2}$. The reason for this difference is that the Landau damping term goes as $\mathrm{x}_{\mathrm{e}} / \mathrm{x}$ for large $x$ (as against $x_{R}^{2} / x^{2}$ for resistive case). To avoid the logarithmic 
singularity at large $x$, we have to introduce a cutoff at the ionLandau resonance point $x=x_{I}$. This explains the $x_{I}$ in the logarithm. Equation (18) leads to the growth rate $(\mathrm{n} / 2) \pi^{-1 / 2}\left(\mathrm{~L}_{\mathrm{n}} / \beta L_{\mathrm{s}}\right)(\mathrm{m} / \mathrm{M})^{1 / 2} \omega_{*} \ln (1 / \sqrt{\beta})$ which is again a significantly large fraction of $\omega_{*}$. Further, in this case also a "tearing" component of magnetic field fluctuation is finite for even modes, electron inertia removing the singularity at $x-0$.

Two conditions necessary for the validity of above analysis were " $11<\omega_{*}$ and $\left|\mathrm{d}^{2}, a \mathrm{x}^{2}\right|$; $>\mathrm{k}_{\mathrm{y}}^{2}$. The two may be combined to give $(\mathrm{n} / \mathrm{m})\left(\mathrm{L}_{\mathrm{n}} / \mathrm{L}_{\mathrm{s}}\right)\left(\mathrm{L}_{\mathrm{n}} / \lambda_{\mathrm{s}}\right)>\beta>\dot{n}^{2}\left(\mathrm{~L}_{\mathrm{n}}^{2} / \mathrm{L}_{\mathrm{s}}^{2}\right)$ where $\mathrm{m}$ is azimuthal mode number and $n$ the integer in Eq. (9). We have also assumed $x_{e}^{2}$, $x_{\mathrm{R}}^{2} \ll x_{\mathrm{A}}^{2} \ll x_{\mathrm{T}}^{2}$ which requires $\omega / \omega_{*}, \nu / \omega_{*} \ll(\beta M / m)$ and $\omega / \omega_{*}>\beta$ respectively, conditions which may be readily satisfied.

We now spernlate on thr consoguenutu of micioscopic magnetic islands of the type generated by this instability, on anomalous transport. The typical width $w$ of an island is given by $\left(\delta B_{x}{ }_{s}{ }^{\prime}\right.$ $\left.k_{y_{0}}{ }_{0}\right)^{1 / 2}$. Using the linear relation between $\delta B_{x}$ and $\delta n_{e}$, we get

$$
\frac{w}{L_{n}} \sim \beta^{1 / 2}\left(\frac{L_{s}}{L_{n}}\right)^{3 / 1}\left(\frac{\lambda s}{L_{n}}\right)^{1 / 2}\left(\frac{\delta n}{n_{0}}\right)^{1 / 2} .
$$

For $L_{s} / L_{n} \sim 15, \lambda_{\mathrm{s}} / L_{n} \cdot 1.0^{-2}, \beta \cdots 10^{-2}, \delta n / n_{0} \sim 10^{-2}$, this gives $\mathrm{w} / \mathrm{L}_{\mathrm{n}} \sim 10^{-2}$. Thus, the size of the island can become comparable to the Larmor radius for the typical density flcutuations observed in toroidal devices. We also know that the distance between mode rational surfaces is given by 


$$
\frac{\Delta r_{s}}{L_{n}} \sim\left(k_{y} \lambda_{s}\right)^{-1} \frac{\lambda_{s}}{L_{n}} \frac{q R}{L_{s}} \simeq\left(k_{y} \lambda_{s}\right)^{-1} 10^{-2}
$$

Thus for $k_{y} \lambda_{s} \sim 1$, we have $w \sim \Delta I_{s} i . e$. , there will be a strong overlap of magnetic islands on neighboring rational surfaces. It is quite likely then that the loss of heat and particles from the "confined" plasma is governed by a parallel flow along newly re-connected field lines. Taking a parallel collisional random walk model along field lines, which are themselves randomly going in and out due to ergodicity arising due to overlapping magnetic islands, one comes up with an energy confinement time $\tau_{E} \sim\left(\nu_{e} / v_{e}^{2}\right)\left(q R L_{n}^{2} / \lambda_{s}^{2}\right)^{2}$ which has the correct order of magnitude for typical tokamaks and also gives the observed scaling $\tau_{E} \sim \mathrm{n}$. 


\section{REFERENCES}

* This work was jointly supported by U. S. Energy Research and Development Administration Contract No. E(11-1)-3073 and by U. S. Air Force office of Scientific Research Contract No. F $44620-75-\mathrm{C}-0037$.

$1_{B \text {. B. Kadomtsev and O. P. Pogutse, Reviews of Plasma Physics }}$ ‥ $24.9(1.970)$.

2A. B. Mikhailovskii, Theory of Plasma Instabilities, (Consultants Bureau, NY, 1974) Vol. 2, p. 62 .

${ }^{3}$ R. J. Catto, A. M. El Nadi, C. S. Liu and M. N. Rosenbluth, Nucl. Fusion 14, 405 (1974).

${ }^{4}$ B. B. Kadomtsev, Plasma Turbulence, (Acadenic Press, Inñdnn, 1965) p. 82 .

${ }^{5}$ H. P. Furth, J. Killeen and M. N. Rosenbluth, Phys. Fluids 6. $459(1963)$.

6I. D. Pearlstein and H. L. Berk, Phys. Rev. Letters 23 , 220 (1969). 